\title{
Pharmacogenomics and its Future Implications in Treatment-resistant Depression
}

Prasad Shetty

\begin{abstract}
The global burden of depression is estimated over 300 million, which is equivalent to $4.4 \%$ of the world's population. However, it is reported that only $50 \%$ of patients receive some form of treatment and most individuals receiving conventional pharmacotherapy fail to achieve and sustain remission. Treatment-resistant depression (TRD) is defined as failure to achieve remission with two or more adequate antidepressants over a sufficient period of time. Treatment-resistant depression is estimated to occur in 10-30\% of patients with major depression, and these patients need a variety of treatment strategies employed. Advances in genetic epidemiology have spurred research investigating the role genetics play in the pathophysiology of depression. Pharmacogenetic testing identifies mutations related to altered expression and functions of genes associated with antidepressant response. For this reason, genetic variants are considered theoretically optimal biomarkers to provide personalized antidepressant treatments and to reduce the proportion of patients who develop TRD. This review aims to understand the genetic mechanisms that contribute to the problem of TRD and the hurdles to its treatment in the context of the Indian population. We focus on the authors' clinical experience in parallel with relevant research articles over the last two decades.

Keywords: Antidepressants, Depression, Genetics, Pharmacotherapy, Treatment resistance.

Indian Journal of Private Psychiatry (2019): 10.5005/jp-journals-10067-0044
\end{abstract}

\section{INTRODUCTION}

The burden of depression is increasing despite advancements in the safety and tolerability of treatments over the past 50 years. ${ }^{1}$ Researchers and clinicians have shifted their view to depression as a chronic and recurrent illness that may not respond to treatment from an earlier opinion of it being an acute illness. At a global level, over 300 million people are estimated to suffer from depression, equivalent to $4.4 \%$ of the world's population. ${ }^{2}$ However, it is reported that only $50 \%$ of them receive some form of treatment. Most individuals receiving conventional pharmacotherapy fail to achieve and sustain remission-a critical determinant of full functional recovery. ${ }^{3}$ Treatment-resistant depression (TRD) is estimated to occur in 10-30\% of patients with major depression, and these patients need a variety of treatment strategies employed. ${ }^{4}$ Treatment-resistant depression is defined as an inadequate response following adequate antidepressant therapy among patients suffering from unipolar depressive disorders. ${ }^{5}$ Gaynes et al. aimed to approach TRD as a dichotomous term - "patients either have or do not have this diagnosis - based on whether they meet a set of threshold criteria, or the second approach considers TRD an illness that falls along a spectrum, with different degrees, or stages, of severity." ${ }^{16}$ In this latter scheme, either patients do not have TRD at all or they have different degrees of TRD severity. ${ }^{6}$ This review aims to understand the genetic mechanisms that contribute to the problem of TRD and the hurdles to its treatment in the context of the Indian population. We focus on the authors' clinical experience in parallel with relevant research articles over the last two decades.

\section{Depression In India}

Several cost-effective interventions have shown favorable recovery and positive outcomes in the care and management of depression. However, in India, the stigma and treatment gap associated with depression is vast. The burden of depression, in terms of disability adjusted life years (DALYs), increased by 67\% between 1990 and
Department of Psychiatry, Pfizer Limited, Mumbai, Maharashtra, India Corresponding Author: Prasad Shetty, Pfizer Limited, Mumbai, Maharashtra, India, Phone: +91 9322605317, e-mail: Prasad.Shetty2@ Pfizer.com

How to cite this article: Shetty P. Pharmacogenomics and its Future Implications in Treatment-resistant Depression. Ind J Priv Psychiatry 2019;13(2):71-76.

Source of support: Nil

Conflict of interest: None

2013. ${ }^{7}$ By 2025, DALYs attributable to depression are projected to rise by roughly 2.6, 11 million (22.5\%) due to population growth and ageing. ${ }^{7}$ As per National Mental Health Survey (NMHS) (2015-2016) in India, 1 in 20 (5.25\%) people over 18 years of age has ever suffered (at least once in their lifetime) from depression amounting to a total of over 45 million persons with depression in $2015 .{ }^{8}$

\section{Pharmacogenetic Variability in Patients}

For many drugs, the optimum dose required to produce the desired effect for safe therapy shows significant variation from one patient to the next. A drug dose within the therapeutic window for the majority of a patient population may prove to be too low or too high for certain patients who may have an atypical dose-response curve for a drug therapeutic effect or adverse effects. ${ }^{9}$ Pharmacogenetic studies in major depressive disorder (MDD) have been performed only in recent years, focusing on candidate genes with possible involvement in the pathophysiology of the disease and in the mechanism of action of antidepressant drugs. ${ }^{10}$ Factors that result in variations in drug response are multifold and complex, and may involve fundamental aspects of human biology, since a drug response directly affects well-being and survival. ${ }^{9}$ Polymorphisms in genes encoding drug targets directly affect target protein function, 
drug-target interaction, or both to produce profound effects on drug response. ${ }^{9}$ Several studies have found genetic variations associated with altered treatment response/efficacy and increased side effect risk. Genetic testing for such variations can help identify which patients are more or less likely to respond to psychotropics and which are likely to experience an increased side effect burden. Incorporation of this information can drive appropriate treatment choices to improve treatment outcomes. ${ }^{11}$ This benefits patients who may be treatment-resistant by enabling the choice of a more effective drug at an appropriate dose, which can potentially decrease the trial and error approach of antidepressant selection. While there are shortcomings of the applications of this approach, further studies in the domain can significantly impact the outcomes for patients with TRD.

\section{Prevalence of Treatment-resistant Depression}

The treatment outcomes and transitional points across the illness course (e.g., response, remission, and relapse) directly influence how TRD is conceptualized. A trial funded by the National Institute of Mental Health known as the Sequenced Treatment Alternatives to Relieve Depression (STAR*D) recruited in over 4,000 patients suffering from depression. The trial showed cumulative remission rates in only half of the patients after trial of two different treatments. However, other estimates point to rates of $60-70 \%$ for incomplete remission. ${ }^{12}$

\section{Management of Treatment-resistant Depression}

Patients diagnosed with MDD are majorly prescribed either selective serotonin reuptake inhibitors (SSRIs) or serotonin-norepinephrine reuptake inhibitors (SNRIs) as the first option. If the patient fails to achieve the adequate response, then the most common approach is to switch the patient to a second antidepressant. The drugs used for augmentation in TRD backed with evidence include lithium (with TCA), bupropion or mirtazapine combination therapy (with SSRI), anticonvulsants, thyroid hormone (with TCA), atypical anticonvulsants, dopamine agonists, pindolol, buspirone, modafinil, stimulants, testosterone, or estrogen. Combining an SSRI and a noradrenergic reuptake inhibitor confers the double effect of antidepressant as an augmentation strategy. The same advantage is offered by either switching to or adding duloxetine or venlafaxine. Venlafaxine has been reported to have a significantly high remission rate (42\%). Desvenlafaxine, a SNRI, is approved for the treatment of adults with MDD. It is the major active metabolite of venlafaxine after metabolism by CYP2D6 and possesses antidepressant activity. Desvenlafaxine has a lower risk of variability in pharmacologic effect resulting from CYP2D6 polymorphisms or drug-drug interactions when coadministered with CYP2D6 substrates or inhibitors. The simple metabolic pathway of desvenlafaxine makes it favorable in avoiding CYP-related pharmacokinetic interactions with other drugs of many therapeutic classes, thus giving desvenlafaxine an edge over other antidepressants in management of TRD.

\section{Factors Associated with Treatment-resistant Depression}

The high percentage of treatment failure or incomplete remission is likely to be a consequence of intrinsic biological and environmental heterogeneity among MDD patients, suggesting that biomarkers of antidepressant response would be useful to guide treatment at the individual level. ${ }^{13}$ This correlates with the clinical observation that MDD is a heterogeneous disease. Treatment-resistant depression patients have some distinctive clinical features such as higher symptom severity, more frequent suicidal risk, and comorbidity with anxiety as compared to non-TRD patients. ${ }^{13}$

Treatment-resistant depression is a result of possible multiple factors. These can broadly be divided into factors related to the illness, factors related to treatment, and patient and environmental factors. Usually, combinations of factors are involved in treatment resistance. ${ }^{14}$ Patients with TRD are reported to experience a higher number of stressful life events, including immigration, death of a family member, interpersonal relationship problems, job loss, financial stress, severe health conditions, and life-threatening situations. ${ }^{15}$

Genetics has been shown to be a significant factor in the variability of responses of medication choices and doses. Pharmacogenetics has become an increasingly important tool for identifying factors like the risk associated with adverse drug reactions or decreased response to standard doses of medications. By identification of such genetic factors, the clinicians may be able to advise the most effective therapy to their patients. The addition of pharmacogenetic testing to routine clinical practice may also be extremely helpful because of the cost reduction associated with the identification of patients who will not respond to expensive drugs or with the identification of patients likely to suffer from severe adverse events. $^{16,17}$

\section{Potential for Pharmacogenetics in Treatment-resistant Depression}

The role of genetics in the pathophysiology of depression is being explored owing to the advances in the genetic epidemiology. These tests identify mutations related to altered expression and functions of genes associated with drug response. These tests have proven to be useful in clinical settings. Antidepressant response was demonstrated to have a relevant genetic component by family studies and more recent approaches such as genome-wide complex trait analysis. ${ }^{18}$ For this reason, genetic variants are considered theoretically optimal biomarkers to provide personalized antidepressant treatments and to reduce the proportion of patients who develop TRD.

\section{Cytochrome P450 Genes}

Cytochromes are the primary enzymes involved in drug metabolism, and cytochrome P450 oxidases are implicated in the oxidative metabolism of several antidepressants. ${ }^{10}$ Different metabolic profiles are caused by the polymorphic nature of cytochrome $\mathrm{P} 450$ genes, which modifies individual drug response. ${ }^{19}$ The genetic metabolic profile is generally useful in predicting side effects, rather than the response to antidepressants. ${ }^{20}$ The functional polymorphisms consist of gene deletions, gene duplications, and deleterious mutations. Additionally, amino acid changes might be introduced, which can, in some cases, change the substrate specificity. ${ }^{19}$ The CYP2D6 and CYP2C19 genes have the highest importance in clinical guidelines out of all the functional polymorphisms. An important aspect of this polymorphism is the copy number variation where multiple functional gene copies of one allele can result in increased drug metabolism and absence of drug response at standard dosage. ${ }^{19}$ Guideline recommendations point to strong or moderate evidence supporting the choice of an antidepressant and dose based on the presence of functional variants in these two CYP450 genes when treatment is a tricyclic antidepressant, sertraline, citalopram, escitalopram, fluvoxamine, or paroxetine. ${ }^{13}$ 


\section{CYP2D6}

The CYP2D6 enzyme is responsible for the metabolism of roughly a quarter of all drugs used to treat depression, and its polymorphism significantly affects the metabolism of about half of these drugs, making it one of the most important polymorphic enzymes in the metabolism of drugs. Genetic variation contributes largely to the interindividual variation in enzyme activity. ${ }^{19}$ Variant alleles of CYP2D6 are classified on the basis of enzymatic activities. There are four classifications of this enzyme based on its metabolizing activity: poor metabolizers (PMs), intermediate metabolizers (IMs), extensive metabolizers (EMs), and ultraextensive metabolizers (UMs). ${ }^{21}$ Poor metabolizers that have polymorphisms resulting in enzyme deactivation produce high concentrations of the parent drug in the plasma. Ultra-extensive metabolizers lead to increased enzyme activity due to gene duplications, resulting in reduced parent drug concentration in the bloodstream. ${ }^{22}$ CYP2D6 is subject to deletions, gene duplications, or multiplications, many clinical laboratories also report copy number variations, but is not known to be induced by substances. ${ }^{23}$ It is highly polymorphic with over 100 known allelic variants and subvariants identified. Associations of imipramine, trimipramine, desipramine, and clomipramine with CYP2D6 genotypes have been reported. ${ }^{23}$ In addition, the impact of CYP2D6 can be found on nortriptyline, a tricyclic antidepressant (TCA), with these genes explaining $21 \%$ of total interindividual variance in oral clearance. ${ }^{23}$ Venlafaxine, an SNRI, has its metabolism influenced by CYP2D6 polymorphism, particularly the O-demethylation phenotype depends on the CYP2D6 genotype. ${ }^{23}$ This isozymes' tolerance is lower to TCAs and venlafaxine as compared to an average tolerance to other antidepressants. ${ }^{23}$

CYP2D6 alleles have been studied in varied geographic and ethnic group settings, and significant differences in allele frequencies have been observed. ${ }^{24}$ In a sample size of 447 from South India, a study conducted by Naveen et al. showed that the CYP2D6*2 allele was the most frequent variant (34.8\%), followed by the ${ }^{*} 10$ allele (10.2\%). ${ }^{22}$ Studies across India have shown variation in frequency of CYP2D6 polymorphism ranging from 2 to $4.8 \%$ across Maharashtra, Andhra Pradesh, Kerala, and Chandigarh. ${ }^{25}$

\section{CYP2C19}

Polymorphisms seen in the CYP2C19 gene affect the metabolism of several classes of drugs, including antidepressants and proton pump inhibitors. ${ }^{19,24}$ The CYP2C19 gene, like CYP2D6, is highly polymorphic and over 30 allelic variants and subvariants have been identified. The CYP2C19 phenotype also affects the pharmacokinetics of antidepressants; the monoamine oxidase inhibitor (MAOI), the tricyclic antidepressants (TCA) amitriptyline and clomipramine, and the selective serotonin reuptake inhibitors (SSRI) sertraline and citalopram are examples of such drugs. ${ }^{19}$ The enzyme activity is expressed at highly variable levels and three different phenotypes are observed: PM, IM, and normal metabolizers (NM). ${ }^{26}$ CYP2C9*2 and CYP2C9*3 are the two most common polymorphisms. ${ }^{9}$ With respect to the ${ }^{*} 17$ allele, patients homozygous for CYP 2 C $19 * 17$ showed $42 \%$ lower geometric escitalopram mean serum concentrations and 5.7-fold higher for defective CYP2C19 alleles, compared with the CYP2C19*1/*1 subgroup. ${ }^{27}$ Moreover, Chinese subjects with two CYP2C19mutated alleles $(* 2, * 3)$ showed higher plasma concentrations and the amitriptyline:nortriptyline ratio at the steady state than those with the wild-type genotype. ${ }^{28}$
Presence of the CYP2C19*2 allele leads to an aberrant splice site whereas the CYP2C19*3 allele produces a premature stop codon. ${ }^{29}$ Adithan et al. evaluated the frequency of CYP2C19 polymorphisms in India and documented the frequency of CYP2C19*1, *2, and *3 alleles as 0.598 (59.8\%), 0.379 (37.9\%), and 0.022 (2.2\%), respectively, for South Indians $(n=112)$ and $0.703(70.3 \%), 0.297(29.7 \%)$, and 0 , respectively, for North Indians $(n=121) .{ }^{29}$ This showed that the prevalence of PMs in South Indians was significantly higher compared with Caucasians and Africans. In addition, the EM activity of CYP2C19 is significantly reduced due to heterozygosity of the Asian population. For instance, two studies on North and South Indian populations reported the frequency of the CYP2C12*2 allele to be 29.7 and $35 \%$, with the CYP2C19*3 allele to be nil and $1 \%$, respectively. ${ }^{30,31}$

\section{CYP3A4}

The most abundant CYP isoenzyme in the liver and small intestine is CYP3A4, which is also involved in biotransformation of approximately $50 \%$ of marketed drugs. ${ }^{32-34}$ Parallelism in racial, ethnical, and geographical distribution due to polymorphism in human xenobiotic metabolizing genes and the ethnic-specific effect of CYP genes is well known. ${ }^{35}$ The Indian population is a vast distinct ethnic group forming one-sixth of the world population. ${ }^{36}$ CYP1A1 and $3 \mathrm{~A} 4$ are within the cytochrome P450 enzyme family with the ability to metabolize endogenous and exogenous substances. ${ }^{37}$ Polymorphisms in these genes as a result of environmental toxins and exposure to hormones have shown association with cancer susceptibility. ${ }^{35,38}$ Thus, higher risk of development of cancer and other related disorders can be tied to ethnic populations with high prevalence of relevant polymorphisms. ${ }^{38}$ Studies in India have shown variation in the frequency of CYP3A4 polymorphism of the *1B allele ranging from $0.6 \%$ to $2 \%$ across Chandigarh, Delhi, and Mumbai. ${ }^{39}$

\section{Other Isoenzymes}

The CYP2C9 genetic polymorphisms cause reduced enzyme activity in Caucasians (approximately $0.7 \%$ of the population). ${ }^{40}$ It also modulates dose-corrected plasma concentrations of fluoxetine and the active moiety (fluoxetine plus norfluoxetine), along with the CYP2D6 gene variants. ${ }^{41,42}$ However, it has not shown effect on plasma concentration of several antidepressants, including TCAs (amitriptyline, clomipramine, doxepin), SSRIs (citalopram, fluvoxamine, paroxetine, sertraline), and others (mirtazapine, venlafaxine). ${ }^{43}$ The highly polymorphic CYP2B6 gene has not been associated with loss of function due to copy number variations or genetic variants, apart from the rare CYP2B6*28 allele. ${ }^{23}$ A summary of antidepressants metabolized by the CYP isoenzymes is presented in Table 1.

\section{Use of Genetic Testing in Psychiatry}

Individual patients carrying CYP2D6*3, - $* 4,-* 5$, or $-* 6$ variants should be given reduced doses of antidepressants to avoid or reduce drug side effects. In this scenario, metabolism of the drugs is the major factor affecting drug response and safety, and genetic variations are well established. ${ }^{9}$ Psychiatrists at the Mayo Clinic have begun to request that the CYP2D6 genotype information be made available before commencing psychiatric drug therapy. ${ }^{44}$ A study has been conducted analyzing the cost-effectiveness and impact on medication adherence in psychiatric patients who 


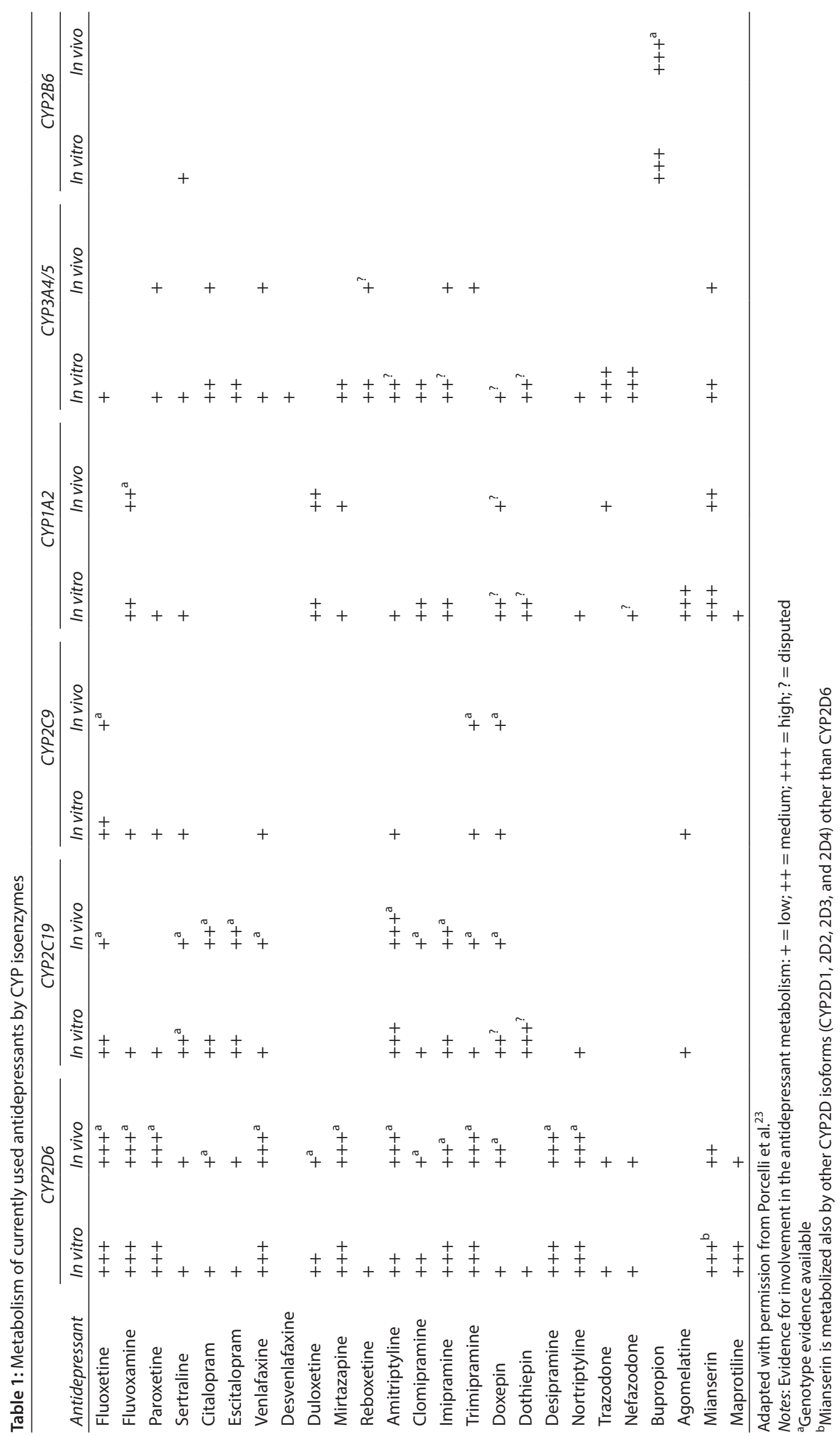


Test-related barriers

- Pharmacogenetic test available in CLIA/CAP-compliant laboratory

- Remembering to order test or identifying patients for whom test is appropriate

- Turnaround time for test results

- Cost of test and potential lack of reimbursement for test

\section{Knowledge barriers}

- Insufficient knowledge of pharmacogenetic data

- Uncertainty about pharmacogenetic test interpretation

- Uncertainty about drug therapy decision based on pharmacogenetic test

\section{Evidence barriers}

- Genetic exceptionalism for genetic and pharmacogenetic tests

- Lack of randomized controlled trial documenting superiority of

pharmacogenetic-guided treatment approach

\section{ELSI barriers}

- Concerns about inclusion of genetic information in the medical record and

potential for genetic discrimination

- Questions about importance of sharing pharmacogenetic findings with family members

- Defining importance of ELSI in pharmacogenetics versus disease genetics

Fig. 1: Summary of barriers to the clinical implementation of pharmacogenetics. Adapted with permission from Johnson (2013). ${ }^{46}$ CAP, College of American Pathologists; CLIA, Clinical Laboratory Improvement, Amendments; ELSI, ethical, legal, and social implications

received a genetic test compared to a matched set of controls. ${ }^{45}$ The abundance of genetic information and DNA sequence data raises the issue of the most efficient and cost-effective way to establish the clinical utility of novel pharmacogenomic information. ${ }^{44}$

There are several reasons that genomic medicine has not moved as fast as hoped. A study by Johnson identified barriers to the clinical implementation of pharmacogenomics (Fig. 1). ${ }^{46}$ In addition to scientific difficulties discussed above, economic, ethical, social, and regulatory issues are also very challenging. A systematic literature review by Berm et al. investigating the cost-effectiveness of pharmacogenetic and pharmacogenomics screening found its application to be mostly cost-effective or cost-saving. ${ }^{47}$ While only a minority of the evaluations assessed the intrinsic value of these tests, there was an increase in studies reporting quality-associated characteristics. ${ }^{47}$ Although the cost of DNA sequencing is dropping dramatically, data on cost-effectiveness of genomic medicine are currently limited. ${ }^{9}$ Pharmacogenomics has the potential to lower costs associated with inappropriate or expensive drug treatments and/or serious adverse drug reactions that may require hospitalization.

\section{Conclusion}

Pharmacogenetic testing for psychiatric disorders is rapidly gaining wide acceptance. Its clinical implementation requires objective, evidence-based guidelines and investment in the infrastructure required to make pharmacogenomic information available to physicians in a cost-effective manner. Clinical utility and cost-effectiveness of genetic testing for psychiatric disorders has been substantiated by a number of reviews and case studies. ${ }^{11}$ Treatment-resistant depression is highly prevalent and is associated with high economic implications and extensive usage of depression-related and general medical services. It poses unique therapeutic challenges and dilemmas in its management. Early identification of TRD will be useful in constructing effective and long-term maintenance treatment strategies. The individualized medicine will enable physicians to prescribe appropriate treatment and dose to achieve maximal therapeutic benefits and minimum adverse effects. ${ }^{9}$ Armed with patient genetic information, clinicians can more quickly identify effective therapies, thus limiting the prolonged suffering and economic burden placed upon many patients with chronic illnesses. Genetic evaluation for patients showing early signs of TRD can be considered as a part of the management strategy. Prospective clinical trials that evaluate the utility and cost-effectiveness of genotyping and individualized medicine are critical in guiding the clinical practice of genetic testing and individualized drug therapy.

\section{References}

1. Murphy JA, Sarris J, Byrne GJ. A review of the conceptualisation and risk factors associated with treatment-resistant depression. Depress Res Treat 2017;2017:4176825. DOI: 10.1155/2017/4176825.

2. Organization WH. Depression and other common mental disorders: global health estimates. World Health Organization; 2017.

3. Mclntyre RS, Filteau M-J, Martin L, et al. Treatment-resistant depression: definitions, review of the evidence, and algorithmic approach. J Affect Disord 2014;156:1-7. DOI: 10.1016/j.jad.2013.10.043.

4. Al-Harbi KS. Treatment-resistant depression: therapeutic trends, challenges, and future directions. Patient Prefer Adherence 2012;6:369. DOI: 10.2147/PPA.S29716.

5. Fava M. Diagnosis and definition of treatment-resistant depression. Biol Psychiatry 2003;53(8):649-659. DOI: 10.1016/S00063223(03)00231-2.

6. Gaynes BN, Asher G, Gartlehner G, et al. Definition of TreatmentResistant Depression in the Medicare Population; 2018. 
7. Charlson FJ, Baxter AJ, Cheng HG, et al. The burden of mental, neurological, and substance use disorders in China and India: a systematic analysis of community representative epidemiological studies. Lancet 2016;388(10042):376-389. DOI: 10.1016/S01406736(16)30590-6

8. WHO, Depression in India Let's Talk India2017 [cited 2019 18th March]. Available from: http://www.searo.who.int/india/depression_in_ india.pdf.

9. Ma Q, Lu AY. Pharmacogenetics, pharmacogenomics, and individualized medicine. Pharmacol Rev 2011;63(2):437-459. DOI: 10.1124/pr.110.003533.

10. Schosser A, Serretti A, Souery D, et al. European Group for the Study of Resistant Depression (GSRD) — where have we gone so far: review of clinical and genetic findings. Eur Neuropsychopharmacol 2012;22(7):453-468. DOI: 10.1016/j.euroneuro.2012.02.006.

11. Gardner KR, Brennan FX, Scott R, et al. The potential utility of pharmacogenetic testing in psychiatry. Psychiatry J 2014;2014:730956. DOI: $10.1155 / 2014 / 730956$

12. Sinyor M, Schaffer A, Levitt A. The sequenced treatment alternatives to relieve depression (STAR* D) trial: a review. Can J Psychiatry 2010;55(3):126-135. DOI: 10.1177/070674371005500303.

13. Fabbri C, Corponi F, Souery D, et al. The genetics of treatmentresistant depression: a critical review and future perspectives. Int J Neuropsychopharmacol 2018;22(2):93-104. DOI: 10.1093/ijnp/pyy024.

14. Parikh RM, Lebowitz BD. Current perspectives in the management of treatment-resistant depression. Dialogues Clin Neurosci 2004;6(1):53.

15. Amital D, Fostick L, Silberman A, et al. Serious life events among resistant and non-resistant MDD patients. J Affect Disord 2008;110(3):260-264. DOI: 10.1016/j.jad.2008.01.006.

16. Roden DM, Wilke RA, Kroemer HK, et al. Pharmacogenomics: the genetics of variable drug responses. Circulation 2011;123(15): 1661-1670. DOI: 10.1161/CIRCULATIONAHA.109.914820.

17. Gupta PD. Pharmacogenetics, pharmacogenomics and ayurgenomics for personalized medicine: a paradigm shift. Indian J Pharm Sci 2015;77(2):135. DOI: 10.4103/0250-474X.156543.

18. Tansey KE, Guipponi M, Hu X, et al. Contribution of common genetic variants to antidepressant response. Biol Psychiatry 2013;73(7): 679-682. DOI: 10.1016/j.biopsych.2012.10.030.

19. Ingelman-Sundberg M, Sim SC, GomezA, et al. Influence of cytochrome P450 polymorphisms on drug therapies: pharmacogenetic, pharmacoepigenetic and clinical aspects. Pharmacol Ther 2007;116(3): 496-526. DOI: 10.1016/j.pharmthera.2007.09.004.

20. Höfer P, Schosser A, Calati R, et al. The impact of Cytochrome P450 CYP1A2, CYP2C9, CYP2C19 and CYP2D6 genes on suicide attempt and suicide risk-a European multicentre study on treatmentresistant major depressive disorder. Eur Arch Psychiatry Clin Neurosci 2013;263(5):385-391. DOI: 10.1007/s00406-012-0375-y.

21. Vegter S, Boersma C, Rozenbaum M, et al. Pharmacoeconomic evaluations of pharmacogenetic and genomic screening programmes. Pharmacoeconomics 2008;26(7):569-587. DOI: 10.2165/00019053-200826070-00005.

22. Naveen AT, Adithan C, Soya SS, et al. CYP2D6 genetic polymorphism in South Indian populations. Biol Pharm Bull 2006;29(8):1655-1658. DOI: $10.1248 / \mathrm{bpb} .29 .1655$.

23. Porcelli S, Fabbri C, Spina E, et al. Genetic polymorphisms of cytochrome P450 enzymes and antidepressant metabolism. Expert Opin Drug Metab Toxicol 2011;7(9):1101-1115. DOI: 10.1517/ 17425255.2011.597740.

24. Hicks JK, Bishop JR, Sangkuhl K, et al. Clinical Pharmacogenetics Implementation Consortium (CPIC) guideline for CYP2D6 and CYP2C19 genotypes and dosing of selective serotonin reuptake inhibitors. Clin Pharmacol Ther 2015;98(2):127-134. DOI: 10.1002/cpt.147.

25. Abraham BK, Adithan C. Genetic polymorphism of CYP2D6. Indian J Pharmacol 2001;33(3):147-169.

26. Siddapuram S, Banerjee R, Tandan M, et al. CYP2C19 polymorphism as a predictor of personalized therapy in South Indian population. J Assoc Physicians India 2011;59:490-493.

27. Rudberg I, Mohebi B, Hermann M, et al. Impact of the ultrarapid CYP2C19* 17 allele on serum concentration of escitalopram in psychiatric patients. Clin Pharmacol Ther 2008;83(2):322-327. DOI: 10.1038/sj.clpt.6100291.

28. Shimoda K, Someya T, Yokono A, et al. The impact of CYP2C19 and CYP2D6 genotypes on metabolism of amitriptyline in Japanese psychiatric patients. J Clin Psychopharmacol 2002;22(4):371-378. DOI: 10.1097/00004714-200208000-00007.

29. Adithan C, Gerard N, Vasu S, et al. Allele and genotype frequency of CYP2C19 in a Tamilian population. Br J Clin Pharmacol 2003;56(3): 331-333. DOI: 10.1046/j.1365-2125.2003.01883.x.

30. Lamba JK, Dhiman RK, Kohli KK. CYP2C19 genetic mutations in North Indians. Clin Pharmacol Ther 2000;68(3):328-335. DOI: 10.1067/ mcp.2000.109365.

31. Jose R, Chandrasekaran A, Sam SS, et al. CYP2C9 and CYP2C19 genetic polymorphisms: frequencies in the south Indian population. Fundam Clin Pharmacol 2005;19(1):101-105. DOI: 10.1111/j.14728206.2004.00307.x.

32. Guengerich FP. Cytochrome P-450 3A4: regulation and role in drug metabolism. Annu Rev Pharmacol Toxicol 1999;39(1):1-17. DOI: 10.1146/annurev.pharmtox.39.1.1.

33. Guengerich FP. Cytochromes P450, drugs, and diseases. Mol Interv 2003;3(4):194. DOI: 10.1124/mi.3.4.194.

34. Nebert DW, Russell DW. Clinical importance of the cytochromes P450. Lancet 2002;360(9340):1155-1162. DOI: 10.1016/S0140-6736(02)112037.

35. Garte S. The role of ethnicity in cancer susceptibility gene polymorphisms: the example of CYP1A1. Carcinogenesis 1998;19(8):1329-1332. DOI: 10.1093/carcin/19.8.1329.

36. Majumder PP, Mukherjee B. Genetic diversity and affinities among Indian populations: an overview. Human population genetics. Springer; 1993; pp. 255-275.

37. Kumar V, Singh S, Yadav CS, et al. CYP1A1 and CYP3A4 polymorphic variations in Delhi population of Northern India. Environ Toxicol Pharmacol 2010;29(2):126-130. DOI: 10.1016/j.etap.2009.12.001.

38. Garte S, Gaspari L, Alexandrie A-K, et al. Metabolic gene polymorphism frequencies in control populations. Cancer Epidemiol Biomarkers Prev 2001;10(12):1239-1248.

39. Umamaheswaran G, Kumar DK, Adithan C. Distribution of genetic polymorphisms of genes encoding drug metabolizing enzymes \& drug transporters-a review with Indian perspective. Indian J Med Res 2014;139(1):27.

40. Kirchheiner J, Müller G, Meineke I, et al. Effects of polymorphisms in CYP2D6, CYP2C9, and CYP2C19 on trimipramine pharmacokinetics. J Clin Psychopharmacol 2003;23(5):459-466. DOI: 10.1097/01. jcp.0000088909.24613.92.

41. LLerena A, Dorado P, Berecz R, et al. Effect of CYP2D6 and CYP2C9 genotypes on fluoxetine and norfluoxetine plasma concentrations during steady-state conditions. Eur J Clin Pharmacol 2004;59(12): 869-873. DOI: 10.1007/s00228-003-0707-y.

42. Scordo MG, Spina E, Dahl ML, et al. Influence of CYP2C9, 2C19 and 2D6 genetic polymorphisms on the steady-state plasma concentrations of the enantiomers of fluoxetine and norfluoxetine. Basic Clin Pharmacol Toxicol 2005;97(5):296-301.DOI: 10.1111/j.1742-7843.2005.pto_194.x.

43. Grasmäder K, Verwohlt PL, Rietschel M, et al. Impact of polymorphisms of cytochrome-P450 isoenzymes 2C9, 2C19 and 2D6 on plasma concentrations and clinical effects of antidepressants in a naturalistic clinical setting. Eur J Clin Pharmacol 2004;60(5):329-336. DOI: 10.1007/ s00228-004-0766-8.

44. Weinshilboum RM, Wang L, ed. Pharmacogenomics: precision medicine and drug response. Elsevier, Mayo Clinic Proceedings; 2017

45. Fagerness J, Fonseca E, Hess GP, et al. Pharmacogenetic-guided psychiatric intervention associated with increased adherence and cost savings. Am J Manag Care 2014;20(5):e146-e156.

46. Johnson JA. Pharmacogenetics in clinical practice: how far have we come and where are we going? Pharmacogenomics 2013;14(7): 835-843. DOI: $10.2217 /$ pgs.13.52.

47. Berm EJ, de Looff M, Wilffert B, et al. Economic evaluations of pharmacogenetic and pharmacogenomic screening tests: a systematic review. second update of the literature. PLoS One 2016;11(1): e0146262. DOI: 10.1371/journal.pone.0146262. 